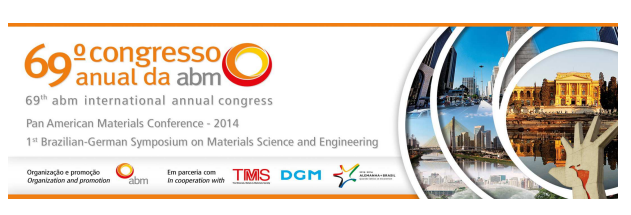

Tema: Materiais cerâmicos, compósitos e poliméricos

\title{
ESTUDO DA DEGRADAÇÃO CAUSADA PELA RADIAÇÃO GAMA EM POLÍMEROS UTILIZADOS EM SERVIÇOS DE RADIOTERAPIA*
}

\author{
Elaine Cristina de Azevedor ${ }^{1}$ \\ Antonio Ramos Netor ${ }^{2}$ \\ Carlos Mauricio Lepienski ${ }^{3}$ \\ Salvador Claro Netor $^{4}$
}

\section{Resumo}

Em serviços de radioterapia são utilizados acessórios confeccionados com diversos tipos de polímeros para auxiliar no posicionamento dos pacientes durante o tratamento e na execução das rotinas de trabalho. A radiação gama pode causar nos polímeros degradação de sua estrutura molecular, dificultar a sua na assepsia e seu reduzir o tempo de vida útil. Neste trabalho é apresentado o estudo da degradação causada pela exposição à radiação gama em polímeros utilizados em serviços de radioterapia. Amostras de policarbonato (PC) e polipropileno (PP), foram expostas a doses de radiação gama de 5 kGy e 25 kGy, sendo analisadas por espectrometria de absorção na região do infravermelho e indentação instrumentada. O PC apresentou indícios de fragilização a partir de $5 \mathrm{kGy}$, reduzindo o tempo de vida útil e o PP apresentou se adequado para o uso em serviços onde haja exposição à radiação gama.

Palavras-chave: Radiação gama; polímero; Indentação instrumentada e infravermelho.

\section{STUDY OF DETERIORATION CAUSED BY GAMMA RADIATION IN POLYMERS USED IN RADIATION THERAPY SERVICES}

\section{Abstract}

In radiotherapy services accessories made of various types of polymers to aid in positioning of patients during treatment and execution of work routines are used. Gamma radiation can cause degradation of polymers in their molecular structure, hamper their in aseptic and reduce its lifetime. In this work the study of degradation caused by exposure to gamma radiation the polymers used in radiotherapy services is presented. Samples of polycarbonate (PC) and polypropylene (PP) were exposed to gamma radiation doses of $5 \mathrm{kGy}$ and $25 \mathrm{kGy}$, and analyzed by atomic absorption spectrometry, infrared and instrumented indentation region. The PC showed signs of embrittlement from $5 \mathrm{kGy}$, reducing the useful life and the PP had appropriate for use in services where there is exposure to gamma radiation.

Keywords: Gamma radiation; Polymer; Indentation and infrared.

\author{
Físico, Dra., professora, PPGEM, UTFPR, Curitiba, PR, Brasil. \\ Tecnólogo em Radiologia, MsC., PPGEB, UTFPR, Curitiba, PR, Brasil. \\ Físico, Dr., professor, PIPE, UFPR, Curitiba, PR, Brasil. \\ Químico, Dr., IQSC, USP, São Carlos, SP, Brasil.
}

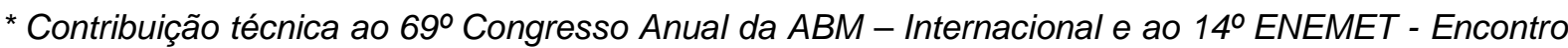
Nacional de Estudantes de Engenharia Metalúrgica, de Materiais e de Minas, 21 a 25 de julho de 2014, São Paulo, SP, Brasil.
} 


\section{INTRODUÇÃO}

Objetos fabricados com polímeros são utilizados em larga escala em serviços hospitalares, devido à facilidade de confecção em diversos formatos, proporcionando uma infinidade de aplicações e terem um baixo custo de fabricação [1]. Em serviços de radioterapia são utilizados posicionadores de membros, como cabeça e pescoço, para garantir a eficiência da execução das irradiações nas regiões de interesse médico, preservando os tecidos sadios [2]. Além destes acessórios, os polímeros são facilmente encontrados em uma variedade de objetos como cadeiras, roupas, mesas, lixeiras, entre outros.

Os simuladores de dose, conhecidos como phantoms, são construídos com materiais poliméricos que absorvem e espalham as radiações ionizantes de forma similar ao corpo humano, possibilitando a avaliação e aplicação de técnicas específicas de tratamento [3]. Estes processos de absorção e espalhamento ocorrem quando as altas energias provenientes das irradiações interagem com a estrutura polimérica, causando excitações e ionizações nas moléculas provocando reações de decomposição e adição nas cadeias [4].

A interação das radiações ionizantes com os polímeros provoca efeitos como cisão e entrecruzamento de cadeias [5], alterando a estrutura molecular e suas propriedades mecânicas e térmicas [6], podendo comprometer a utilização do material nestes ambientes devido a efeitos de endurecimento e fragilização dos materiais, diminuindo a vida útil e gerando custos na reposição destes. A região superficial, onde há a ocorrência dos primeiros efeitos das radiações [4], pode ser afetada de forma que os procedimentos padrões de assepsia adotados nos serviços médicos não tenham eficiência garantida.

Alterações na estrutura química dos polímeros podem ser detectadas através da espectrometria de absorção da região do infravermelho, a partir da análise de espectros formados por picos de intensidade de absorção em função dos números de onda da região do infravermelho [7].

A indentação instrumentada fornece resultados de dureza e módulo elástico das amostras, podendo detectar alterações que provocam fragilização do material [8], reduzindo seu tempo de vida útil.

Este trabalho tem como objetivo avaliar os efeitos da radiação gama nos polímeros com exposição a doses normalmente praticadas em serviços de radioterapia, de forma a simular sua utilização na rotina comum dos serviços ( $5 \mathrm{kGy}$ ) e a dose de esterilização do material (25 kGy).

\section{MATERIAIS E MÉTODOS}

\subsection{Amostras de Polímero}

As amostras de PC, PMMA, PP foram fornecidas pela empresa Nital

\subsection{Irradiação}

A irradiação gama em doses de $5 \mathrm{kGy}$ e $25 \mathrm{kGy}$ foram realizadas pela empresa Embrarad - Cotia-SP, com uma fonte industrial de cobalto-60 MDS Nordion's JS9600.

\footnotetext{
* Contribuição técnica ao $69^{\circ}$ Congresso Anual da ABM - Internacional e ao 14ํㅡㄹ ENEMET - Encontro Nacional de Estudantes de Engenharia Metalúrgica, de Materiais e de Minas, 21 a 25 de julho de 2014, São Paulo, SP, Brasil.
} 


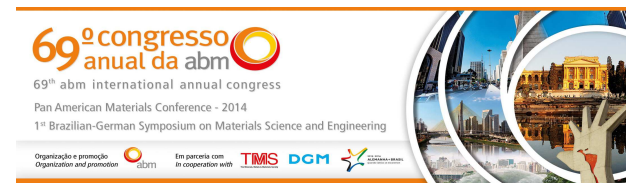

\subsection{Espectroscopia de Absorção na Região do Infravermelho}

Para as caracterizações das amostras, a partir dos espectros de absorção na região do infravermelho, foi usado um espectrômetro infravermelho VARIAN, Modelo FT-IR 640-IR, com resolução de $4 \mathrm{~cm}^{-1}$, faixa de operação de número de onda entre 11000 $\mathrm{cm}^{-1}$ e $735 \mathrm{~cm}^{-1}$, com suporte de ATR.

\subsection{Indentação Instrumentada}

A indentação instrumentada foi utilizada para medir a dureza e o módulo elástico, usando o método de Oliver-Pharr [9] com a ponta Berkovich. As cargas aplicadas variaram de 0,1 a $400 \mathrm{nN}$, utilizando um Nanoindenter XP MTS System.

\section{RESULTADOS E DISCUSSÃO}

\subsection{Policarbonato}

A Figura 1 mostra os espectros das amostras de PC não irradiada e irradiada com 5kGy.

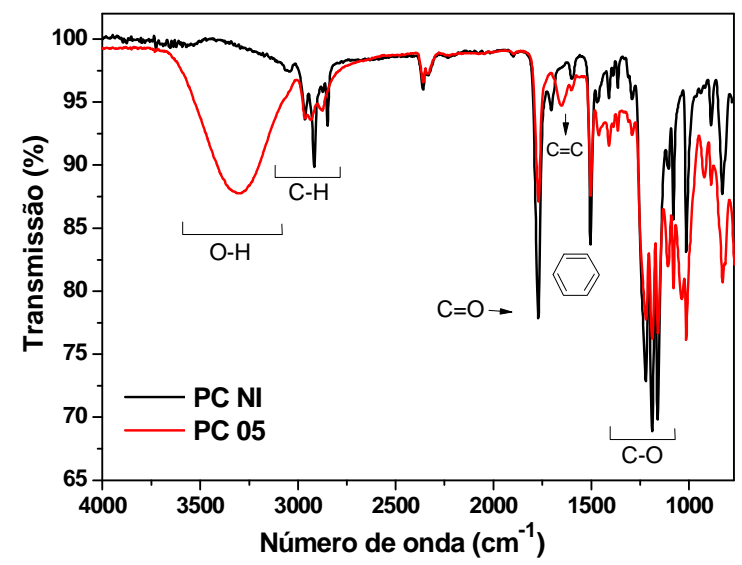

Figura 1 - Espectros das Amostras de PC Não Irradiada e Irradiada com 5 kGy

É possível identificar um forte pico em $1774 \mathrm{~cm}^{-1}$ relativo ao grupo carbonila $(C=O)$, porém os picos mais fortes de absorção de infravermelho no PC ocorrem nos estiramentos das ligações $\mathrm{C}-\mathrm{O}$ sendo possíveis visualizar 3 picos em $1222 \mathrm{~cm}^{-1}$, $1188 \mathrm{~cm}^{-1}$ e $1161 \mathrm{~cm}^{-1}$ e outro pico, menos intenso, em $1015 \mathrm{~cm}^{-1}$. Aos anéis aromáticos da estrutura química atribui-se o pico mais forte em $1510 \mathrm{~cm}^{-1}$. Os estiramentos simétricos e assimétricos do grupo $\mathrm{C}-\mathrm{H}$ são vistos na região entre $2970 \mathrm{~cm}^{-1}$ e $2850 \mathrm{~cm}^{-1}$.

$\mathrm{Na}$ sobreposição destes espectros é possível destacar o aparecimento de uma banda de absorção com pico em $3305 \mathrm{~cm}^{-1}$, referente grupo $\mathrm{OH}$; diminuição da intensidade de absorção dos picos referentes ao grupo $\mathrm{CH}$, entre $2970 \mathrm{~cm}^{-1}$ e 2850 $\mathrm{cm}^{-1}$; diminuição intensidade de absorção dos picos em $1774 \mathrm{~cm}^{-1}$ e $1704 \mathrm{~cm}^{-1}$, referentes ao grupo $\mathrm{C}=\mathrm{O}$; diminuição intensidade de absorção do pico em $1510 \mathrm{~cm}^{-1}$ referente aos anéis aromáticos; diminuição da intensidade de absorção dos picos em $1222 \mathrm{~cm}^{-1}, 1188 \mathrm{~cm}^{-1}$ e $1161 \mathrm{~cm}^{-1}$, do grupo das ligações $\mathrm{C}-\mathrm{O}$; aparecimento de um pico em $1653 \mathrm{~cm}^{-1}$ e $923 \mathrm{~cm}^{-1}$ indicando a formação de ligações $\mathrm{C}=\mathrm{C}$, possivelmente devido à quebra das ligações do grupo $\mathrm{C}-\mathrm{H}$.

* Contribuição técnica ao $69^{\circ}$ Congresso Anual da ABM - Internacional e ao 14ํㅡㄹ ENEMET - Encontro Nacional de Estudantes de Engenharia Metalúrgica, de Materiais e de Minas, 21 a 25 de julho de 2014, São Paulo, SP, Brasil. 


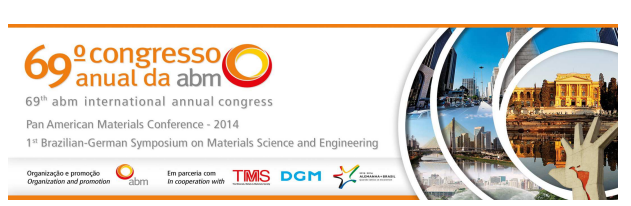

Os valores de dureza são maiores na amostra irradiada quando comparado com a amostra não irradiada, possivelmente devido ao entrecruzamento de cadeias.

As dispersões dos valores de dureza são maiores na superfície do material. As dispersões dos valores de módulo elástico, diferentemente da amostra não irradiada, mantêm-se constante em toda a profundidade medida, possivelmente a amostra sofreu tensão provocando aumento de rugosidade.

Os gráficos de dureza e módulo elástico em função da profundidade de penetração para o PC não irradiado e irradiado com 25 kGy são apresentados nas Figuras 4a e 4b.

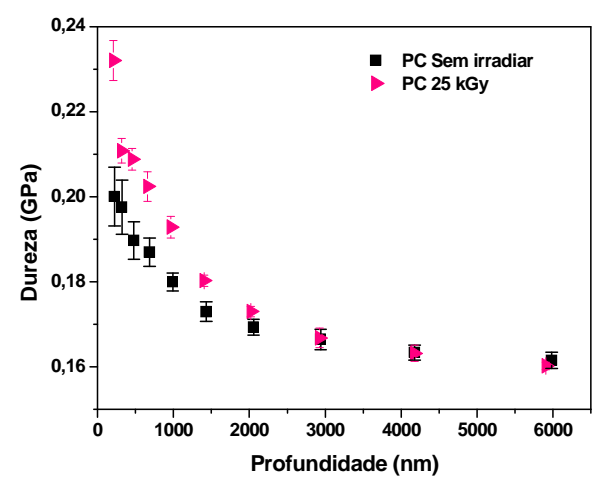

(a)

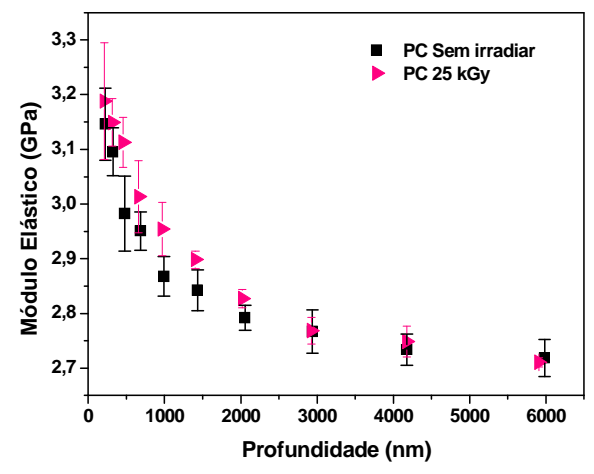

(b)

Figura 4 - (a) Curvas de Dureza em Função da Profundidade de Penetração do PC Não Irradiado e Irradiado com 25 kGy (b) Curvas de Módulo Elástico em Função da Profundidade de Penetração do PC Não Irradiado e Irradiado com 25 kGy

$\mathrm{Na}$ amostra irradiada com 25 kGy o valor da dureza é de 0,23 GPa e o módulo elástico é de $3,18 \mathrm{GPa}$, para a profundidade de penetração de $200 \mathrm{~nm}$. Na profundidade de $6000 \mathrm{~nm}$, a dureza é 0,16 GPa e o modulo elástico é 2,70 GPa.

Os valores de dureza e módulo elástico foram maiores até a profundidade de 3000nm. Após esta profundidade os valores de dureza e módulo elástico são semelhantes aos valores da amostra não irradiada, indicando que o material irradiado foi mais afetado na região superficial próxima.

As dispersões dos valores também são maiores nas profundidades iniciais, possivelmente devido ao aumento da rugosidade do material na superfície.

\subsection{Polipropileno}

A Figura 5 mostra os espectros das amostras de PP não irradiada e irradiada com $5 \mathrm{kGy}$.

\footnotetext{
* Contribuição técnica ao 69ำ Congresso Anual da ABM - Internacional e ao 14ํㅡㄹ ENEMET - Encontro Nacional de Estudantes de Engenharia Metalúrgica, de Materiais e de Minas, 21 a 25 de julho de 2014, São Paulo, SP, Brasil.
} 

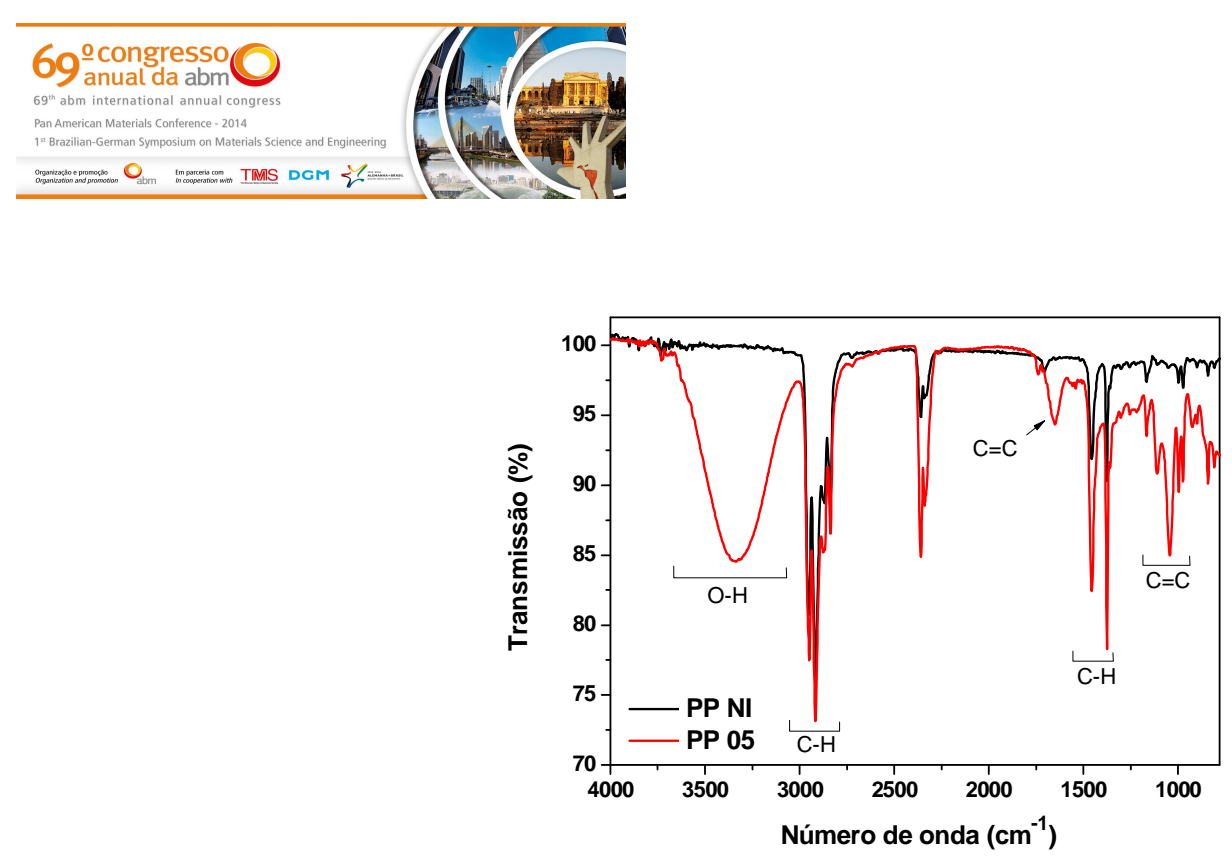

Figura 5 - Espectros das Amostras de PP Não Irradiada e Irradiada com 5 kGy

É possível identificar os picos característicos do PP referentes aos estiramentos simétricos e assimétricos do grupo $\mathrm{C}-\mathrm{H}$ entre $2870 \mathrm{~cm}^{-1}$ e $2950 \mathrm{~cm}^{-1}$, e às deformações simétricas e assimétricas, também do grupo $\mathrm{C}-\mathrm{H}$, nos picos $1455 \mathrm{~cm}^{-1}$ e $1372 \mathrm{~cm}^{-1}$.

Quando comparado com o espectro da amostra irradiada com 5 kGy é possível destacar o aparecimento de uma banda de absorção com pico $3336 \mathrm{~cm}^{-1}$, referente grupo O-H; aparecimento de um pico de baixa intensidade na região de $1647 \mathrm{~cm}^{-1} \mathrm{e}$ outros picos na região entre $1164 \mathrm{~cm}^{-1}$ e $926 \mathrm{~cm}^{-1}$ atribuídos à deformações axiais nas ligações do grupo $\mathrm{C}=\mathrm{C}$, possivelmente devido à quebra de ligações terminais, que favorecem a formação deste grupo; aumento da intensidade de absorção nos picos em $1455 \mathrm{~cm}^{-1}$ e $1372 \mathrm{~cm}^{-1}$ referentes ao grupo $\mathrm{C}-\mathrm{H}$.

A Figura 6 mostra os espectros das amostras de PP não irradiada e irradiada com 25 kGy.

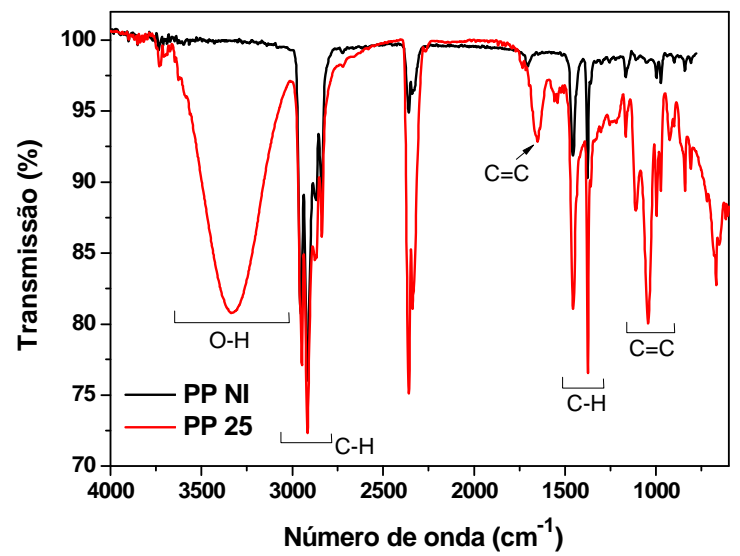

Figura 6 - Espectros das Amostras de PP Não Irradiada e Irradiada com 25 kGy

A análise do espectro da amostra irradiada com $25 \mathrm{kGy}$, mostra que os mesmos picos apresentados no espectro da amostra irradiada com $5 \mathrm{kGy}$ continuam presentes, somente aumentaram suas intensidades de absorção e sem o aparecimento de novos picos.

Sinha [11], estudou efeitos de doses na ordem de 100 kGy e da mesma forma identificou o aparecimento da banda atribuída aos estiramentos do grupo $\mathrm{O}-\mathrm{H}$ e relatou a formação de picos de absorção atribuídos ao grupo $\mathrm{C}=\mathrm{O}$.

Os gráficos de dureza e módulo elástico em função da profundidade de penetração para o PP não irradiado e irradiado com 5 kGy são apresentados na Figura 7a e 7b.

* Contribuição técnica ao 69 Congresso Anual da ABM - Internacional e ao 14ํㅡㄹ ENEMET - Encontro Nacional de Estudantes de Engenharia Metalúrgica, de Materiais e de Minas, 21 a 25 de julho de 2014, São Paulo, SP, Brasil. 


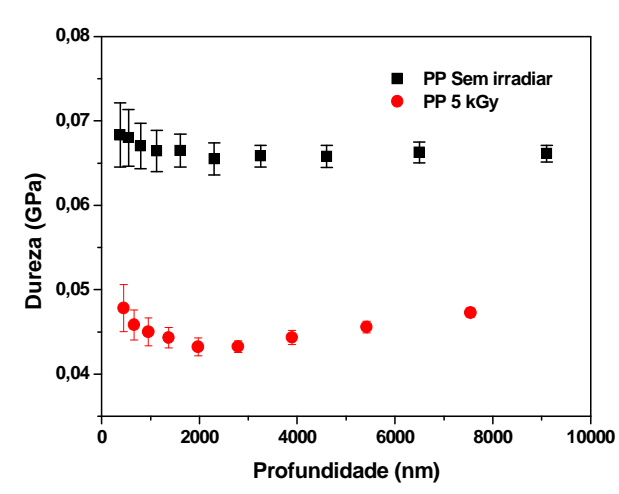

(a)

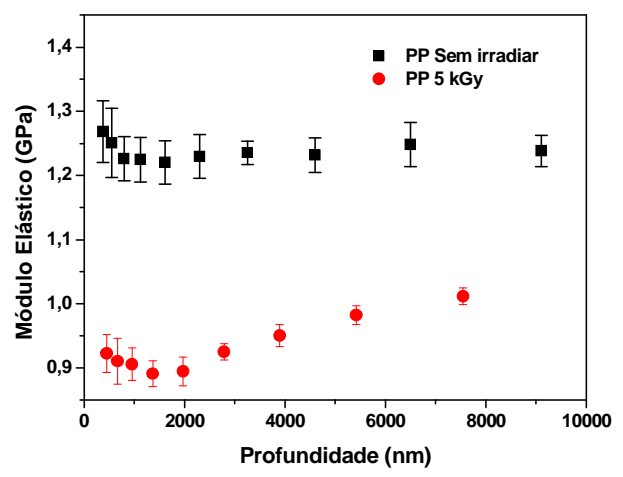

(b)

Figura 7. (a) Curvas de Dureza em Função da Profundidade de Penetração do PP Não Irradiado e Irradiado com 5 kGy (b) Curvas de Módulo Elástico em Função da Profundidade de Penetração do PP Não Irradiado e Irradiado com 5 kGy

$\mathrm{Na}$ amostra irradiada com 5 kGy o valor da dureza é de 0,048 GPa e o módulo elástico é de 0,922 $\mathrm{GPa}$, para a profundidade de penetração de $440 \mathrm{~nm}$. $\mathrm{Na}$ profundidade de $7600 \mathrm{~nm}$ a dureza é $0,047 \mathrm{GPa}$ e o modulo elástico é 1,011 GPa.

Os valores medidos são menores do que os valores medidos na amostra não irradiada, o que indica possíveis processos de cisão de cadeias. Após $2000 \mathrm{~nm}$ os valores são maiores do que nas profundidades iniciais, porém continuam menores quando comparados com a amostra não irradiada.

Os gráficos de dureza e módulo elástico em função da profundidade de penetração para o PP não irradiado e irradiado com 25 kGy são apresentados nas Figuras 8a e $8 b$.

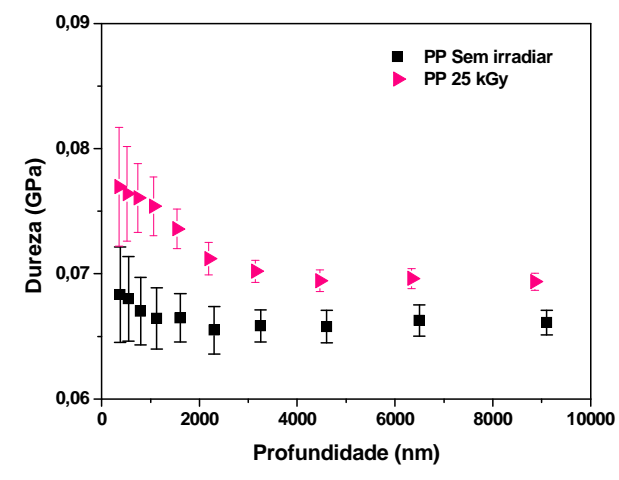

(a)

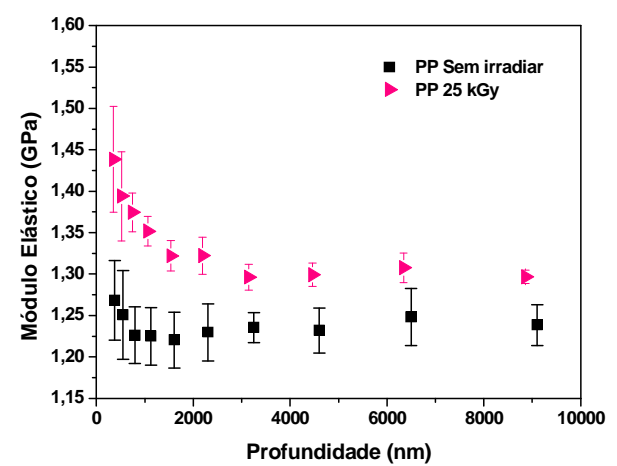

(b)

Figura 8. (a) Curvas de Dureza em Função da Profundidade de Penetração do PP Não Irradiado e Irradiado com 25 kGy (b) Curvas de Módulo Elástico em Função da Profundidade de Penetração do PP Não Irradiado e Irradiado com 25 kGy.

$\mathrm{Na}$ amostra irradiada com 25 kGy o valor da dureza é de 0,077 GPa e o módulo elástico é de $1,440 \mathrm{GPa}$, para a profundidade de penetração de $350 \mathrm{~nm}$. $\mathrm{Na}$ profundidade de $9000 \mathrm{~nm}$, a dureza é 0,069 GPa e o modulo elástico é 1,297 GPa.

Os valores medidos são maiores do que os valores medidos na amostra não irradiada, possivelmente devido ao entrecruzamento de cadeias em toda a profundidade estudada.

As dispersões dos valores da dureza e do módulo elástico são maiores nas profundidades iniciais, indicando aumento de rugosidade do material.

\footnotetext{
* Contribuição técnica ao 69ำ Congresso Anual da ABM - Internacional e ao 14ํㅡㄹ ENEMET - Encontro Nacional de Estudantes de Engenharia Metalúrgica, de Materiais e de Minas, 21 a 25 de julho de 2014, São Paulo, SP, Brasil.
} 


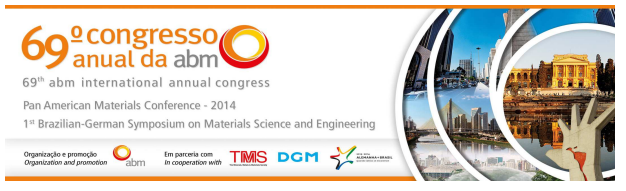

\section{CONCLUSÃO}

Este trabalho avaliou os efeitos da radiação gama nos polímeros com exposição a doses normalmente praticadas em serviços de radioterapia, de forma a simular sua utilização na rotina comum dos serviços.

Dos polímeros estudados, o PC mostrou-se ser o menos indicado para o uso em locais com exposição à radiação gama. Com dose de 5 kGy foi constatado o aumento na dureza e no módulo elástico, possivelmente por entrecruzamento de cadeias, fragilizando o material e diminuindo seu tempo de vida útil. A espectrometria por infravermelho detectou quebra de ligações em todas as doses, o que pode causar a eliminação de compostos voláteis nocivos.

O PP também se mostrou adequado para utilização em serviços de radioterapia. A indentação instrumentada identificou indícios de fragilidade com dose de 25 kGy, devido aos possíveis processos de entrecruzamento de cadeia, tendo assim seu tempo de vida útil. $O$ infravermelho identificou principalmente processos oxidativos superficiais.

\section{Agradecimentos}

Gostaríamos de agradecer ao CNPQ, a CAPES e a Fundação Araucária pelo auxilio financeiro aos laboratórios envolvidos.

\section{REFERÊNCIAS}

1 Canevarolo SV. Ciência dos Polímeros: um texto básico para tecnólogos e engenheiros. São Paulo: Editora Artliber, 2006.

2 Loch CP, Lima MFS. Estudo da degradação dos imobilizadores utilizados em pacientes submetidos à radioterapia de cabeça e pescoço. Matéria (Rio J.), vol. 15, n. 3, Rio de Janeiro, 2010.

3 Scaff LA. Física da Radioterapia. São Paulo: Editora Savier, 1997.

4 Charlesby A. Atomic Radiation and Polymer. London: Editora Pergamom, 1960.

5 Kircher JF, Bowman RE. Effects of Radiation on Materials and Components. New York: Editora Reinholds, 1964.

6 Schnabel W. Polymer Degradation - Principles and Practical Applications. Munich: Ed. Hanser International, 1981.

7 Silverstein RM, Webster FX, Kiemle DJ. Spectrometric Identification of Organic Compounds, 7 ed. New Jersey: Ed. Wiley, 2005.

8 Fischer-Cripps AC. A simple phenomenological approach to nanoindentation creep. Materials Science Engineering, 385, 74-82, 2004.

9 Oliver WC, Pharr GM. Improved technique for determining hardness and elastic modulus using load and displacement sensing indentation experiments, Journal of Materials Research, 1992; 7(6): 1564-1580.

10 Melo NS. Comportamento mêcanico do policarbonato exposto à radiação gama. 2004. 151 f. Dissertação (Mestrado) - Programa de Pós-Graduação em Ciência dos Materiais, Instituto Militar de Engenharia. Rio de Janeiro, 2004.

11 Sinha D. Structural Modifications of Gamma Irradiated Polymers: An FT-IR Study. Advances in Applied Science Research, 2010; 3(3): 1365-1371, Índia.

\footnotetext{
* Contribuição técnica ao $69^{\circ}$ Congresso Anual da ABM - Internacional e ao 14ํㅡㄹ ENEMET - Encontro Nacional de Estudantes de Engenharia Metalúrgica, de Materiais e de Minas, 21 a 25 de julho de 2014, São Paulo, SP, Brasil.
} 